\title{
Lattice dynamics of endotaxial silicide nanowires
}

\author{
J. Kalt, ${ }^{1,2}$ M. Sternik $\odot,{ }^{3}$ B. Krause $\odot,{ }^{2}$ I. Sergueev, ${ }^{4}$ M. Mikolasek, ${ }^{5}$ D. Merkel,,${ }^{6,7}$ D. Bessas $\odot,{ }^{5}$ O. Sikora $\odot,{ }^{3}$ T. Vitova, \\ J. Göttlicher, ${ }^{2}$ R. Steininger, ${ }^{2}$ P. T. Jochym $\odot,{ }^{3}$ A. Ptok $\odot,{ }^{3}$ O. Leupold, ${ }^{4}$ H.-C. Wille, ${ }^{4}$ A. I. Chumakov, ${ }^{5}$ P. Piekarz ${ }^{3}{ }^{3}$ \\ K. Parlinski, ${ }^{3}$ T. Baumbach, ${ }^{1,2}$ and S. Stankov (1,2,* \\ ${ }^{1}$ Laboratory for Applications of Synchrotron Radiation, Karlsruhe Institute of Technology, D-76131 Karlsruhe, Germany \\ ${ }^{2}$ Institute for Photon Science and Synchrotron Radiation, Karlsruhe Institute of Technology, D-76344 Eggenstein-Leopoldshafen, Germany \\ ${ }^{3}$ Institute of Nuclear Physics, Polish Academy of Sciences, PL-31342 Kraków, Poland \\ ${ }^{4}$ Deutsches Elektronen-Synchrotron, D-22607 Hamburg, Germany \\ ${ }^{5}$ ESRF - The European Synchrotron, F-38000 Grenoble, France \\ ${ }^{6}$ Institute for Particle and Nuclear Physics, Wigner Research Centre for Physics, Hungarian Academy of Sciences, H-1525 Budapest, Hungary \\ ${ }^{7}$ Centre for Energy Research, H-1121 Budapest, Hungary \\ ${ }^{8}$ Institute for Nuclear Waste Disposal, Karlsruhe Institute of Technology, D-76344 Eggenstein-Leopoldshafen, Germany
}

(Received 1 August 2020; revised 30 September 2020; accepted 15 October 2020; published 10 November 2020)

Self-organized silicide nanowires are considered as building blocks of future nanoelectronics and have been intensively investigated. In nanostructures, the lattice vibrational waves (phonons) deviate drastically from those in bulk crystals, which gives rise to anomalies in thermodynamic, elastic, electronic, and magnetic properties. Hence a thorough understanding of the physical properties of these materials requires a comprehensive investigation of the lattice dynamics as a function of the nanowire size. We performed a systematic lattice dynamics study of endotaxial $\mathrm{FeSi}_{2}$ nanowires, forming the metastable, surface-stabilized $\alpha$ phase, which are in-plane embedded into the $\mathrm{Si}(110)$ surface. The average widths of the nanowires ranged from 24 to $3 \mathrm{~nm}$, and their lengths ranged from several micrometers to about $100 \mathrm{~nm}$. The Fe-partial phonon density of states, obtained by nuclear inelastic scattering, exhibits a broadening of the spectral features with decreasing nanowire width. The experimental data obtained along and across the nanowires unveiled a pronounced vibrational anisotropy that originates from the specific orientation of the tetragonal $\alpha-\mathrm{FeSi}_{2}$ unit cell on the $\mathrm{Si}(110)$ surface. The results from first-principles calculations are fully consistent with the experimental observations and allow for a comprehensive understanding of the lattice dynamics of endotaxial silicide nanowires.

DOI: 10.1103/PhysRevB.102.195414

\section{INTRODUCTION}

Metallic silicides constitute an important part of current microelectronics, serving as Schottky barriers and ohmic contacts, gate electrodes, local interconnects, and diffusion barriers [1-3]. The enormous degree of miniaturization of present-day integrated circuits imposes severe restrictions on the spatial dimensions of these components. New materials and configurations are constantly researched for nanoelectronic applications, and endotaxial silicide nanowires (NWs) self-organized on the $\mathrm{Si}$ surface have been considered as promising candidates [4]. The endotaxial mechanism implies the formation of in-plane unidirectionally aligned, highaspect-ratio NWs grown partially into the substrate [5]. These nanostructures are readily integrated with $\mathrm{Si}$ technology and exhibit high crystal phase purity and thermal stability, sharp interfaces, and Schottky barrier heights, which are tunable by the choice of silicide material [6]. $\mathrm{FeSi}_{2}$ is a particularly attractive silicide since it exhibits several crystal phases, namely, the room-temperature stable semiconducting $\beta$ phase, high-temperature metallic $\alpha$ phase, and surface-stabilized metallic $s$ and $\gamma$ phases [4]. Due to the very small lattice mismatch with certain crystallographic planes of $\mathrm{Si}$, the tetragonal

\footnotetext{
*svetoslav.stankov@kit.edu
}

$\alpha-\mathrm{FeSi}_{2}$ can also be stabilized at room temperature in epitaxial nanostructures on the $\mathrm{Si}(001), \operatorname{Si}(110)$ and $\mathrm{Si}(111)$ surfaces. However, the reports about the crystal structure of $\mathrm{FeSi}_{2} \mathrm{NWs}$ formed on $\mathrm{Si}(110)$ remain contradictory, spanning cubic $(s$ or $\gamma)$ [7-9] and tetragonal $(\alpha)$ [10] phases.

When the dimensions of nanostructures approach the characteristic phonon mean free paths (from several nanometers up to micrometers), the phonon dispersions and the phonon density of states (PDOS), which characterize the lattice dynamics of a material, begin to deviate from those of the bulk counterparts. These deviations imply significant modifications of thermodynamic and elastic properties, which are directly related to the lattice dynamics, such as lattice heat capacity, vibrational entropy, mean force constants, sound velocity, and thermal conductivity. They also lead to an enhanced electron-phonon, spin-phonon, and phonon-phonon scattering at surfaces and interfaces [11]. In conjunction with a possible emergence of phonon quantum phenomena at very small dimensions [12], these effects could significantly deteriorate the electron and spin transport in nanoscale interconnects [13-15].

The phonon dispersions and PDOS of one-dimensional (1D) nanostructures have been subjects of intensive theoretical studies, predicting features that differ significantly from those in the three-dimensional (3D) counterparts. The most 
prominent effects are confined bands and band gaps, acoustic modes with nonzero frequencies at $q=0$ ( $q$ is the phonon wave vector), nonlinear dispersion for small $q$, and a complex displacement field [16-24]. Consequently, anomalies in thermal conductivity [25-31] and electron-phonon interactions [32-35] were predicted and strategies for their tailoring in NWs were suggested [36].

Unlike the intensive theoretical studies, the experimental reports on lattice dynamics of NWs remain scarce. Confinement of optical phonons in $\mathrm{Si}$ [37-41] and III-V [42] NWs were studied by Raman spectroscopy. Resonant and propagative coherent acoustic phonon modes were investigated by time-resolved spectroscopy with visible light [43] and $\mathrm{x}$ rays [44]. Using Brillouin-Mandelstam light-scattering spectroscopy, surprisingly strong confinement effects in the acoustic phonon dispersions were observed in individual GaAs NWs with diameters exceeding by an order of magnitude the phonon mean free path [45]. Employing nuclear inelastic scattering (NIS) on the ${ }^{125} \mathrm{Te}$ isotope, the Te partial PDOS of a $\mathrm{Bi}_{2} \mathrm{Te}_{3} \mathrm{NW}$ array with an average NW diameter of $56 \mathrm{~nm}$ was determined, unveiling a reduction of the speed of sound by $7 \%$ compared to the bulk material [46]. By application of the same experimental technique on the ${ }^{119} \mathrm{Sn}$ resonance, a correlation between the lattice softening in Sn NWs with a diameter between 100 and $18 \mathrm{~nm}$ and an increase of the critical temperature of the superconducting state was established [47]. Despite their potential applications in nanoelectronics, the lattice dynamics of endotaxial silicide NWs remains unexplored.

Here we present a systematic lattice dynamics study of endotaxial $\mathrm{FeSi}_{2} \mathrm{NW}$ s formed on $\mathrm{Si}(110)$ for a large range of sizes. The Fe-partial PDOS exhibits a broadening of the spectral features with decreasing NW width. The experimental data obtained along and across the NWs unveil a pronounced vibrational anisotropy that originates from the specific orientation of the tetragonal $\alpha-\mathrm{FeSi}_{2}$ unit cell on the $\mathrm{Si}(110)$ surface and is fully consistent with the results from first-principles calculations.

\section{EXPERIMENTAL AND THEORETICAL DETAILS}

Endotaxial $\mathrm{FeSi}_{2} \mathrm{NWs}$ were grown on the $16 \times 2$ reconstructed $\mathrm{Si}(110)$ surface under UHV conditions $(P<1 \times$ $\left.10^{-8} \mathrm{~Pa}\right)$. The substrates were degassed in UHV at $650^{\circ} \mathrm{C}$ for $4 \mathrm{~h}$, followed by the removal of the native $\mathrm{SiO}_{2}$ layer by heating two times to $1250^{\circ} \mathrm{C}$ for $30 \mathrm{~s}$. Subsequently, the $\mathrm{Si}(110)$ surface was stabilized at a growth temperature $T_{G}$ and a certain amount $\theta_{\mathrm{Fe}}$ of high-purity iron, enriched to $96 \%$ in the Mössbauer-active isotope ${ }^{57} \mathrm{Fe}$, was deposited. The Fe coverage was controlled by a quartz oscillator with an accuracy of $10 \%$ and is given in monolayer (ML) units, with $1 \mathrm{ML}=4.78 \times 10^{14} \mathrm{Fe}$ atoms $/ \mathrm{cm}^{2}$. Details of the growth and experimental conditions used for the investigated samples, hereafter referred to as S1-S7, are summarized in Table I. All measurements described in the following were conducted at room temperature. The crystal structure of the samples was investigated with reflection high-energy electron diffraction (RHEED); the surface topography was determined by noncontact atomic force microscopy (AFM) in an Omicron Large Sample scanning probe microscope connected to the
TABLE I. Overview of the investigated samples. $\theta_{\mathrm{Fe}}$ stands for the deposited amount of ${ }^{57} \mathrm{Fe}, T_{G}$ for the growth temperature, and $\bar{w}$ for the average NW width. The last column denotes whether the sample was capped with Si or measured in situ during the NIS experiment.

\begin{tabular}{lrcrc}
\hline \hline Sample & $\theta_{\mathrm{Fe}}[\mathrm{ML}]$ & $T_{G}\left[{ }^{\circ} \mathrm{C}\right]$ & $\bar{w}[\mathrm{~nm}]$ & NIS exp. \\
\hline S1 & $3 \pm 0.3$ & $825 \pm 20$ & $24 \pm 7$ & Si cap \\
S2 & $6 \pm 0.6$ & $700 \pm 10$ & $18 \pm 5$ & Si cap \\
S3 & $2 \pm 0.2$ & $700 \pm 10$ & $10 \pm 3$ & Si cap \\
S4 & $2 \pm 0.2$ & $700 \pm 10$ & $11 \pm 3$ & In situ \\
S5 & $1.5 \pm 0.2$ & $600 \pm 10$ & $4 \pm 1$ & Si cap \\
S6 & $1 \pm 0.1$ & $600 \pm 10$ & $3 \pm 1$ & Si cap \\
S7 & $4 \pm 0.4$ & $825 \pm 20$ & $26 \pm 7$ & - \\
\hline \hline
\end{tabular}

UHV cluster. Samples S1, S2, S3, S5, and S6 were subsequently capped with $4 \mathrm{~nm}$ of amorphous $\mathrm{Si}$ sputtered at room temperature in a chamber [48] with a base pressure of $P<1 \times 10^{-6} \mathrm{~Pa}$ also connected to the UHV cluster. The flux of the sputter gas Ar was $0.8 \mathrm{sccm}$, corresponding to a pressure of $0.36 \mathrm{~Pa}$.

The local crystal structure of the NWs in S2, S6, and S7 was investigated by $\mathrm{Fe} K$-edge x-ray absorption spectroscopy at the SUL-X beamline of the synchrotron radiation source KARA at the Karlsruhe Institute of Technology. After calibration with an $\alpha$-Fe metal foil to the $\mathrm{Fe} K$ edge at $7112 \mathrm{eV}$, the fluorescence emission of the samples was recorded up to $k=14 \AA^{-1}$. A beam-to-sample-to-detector geometry of $45^{\circ} / 45^{\circ}$ was applied, using a collimated $\mathrm{x}$-ray beam of about $0.8 \times 0.8 \mathrm{~mm}$, or a focused $\mathrm{x}$-ray beam with $0.35 \times 0.15 \mathrm{~mm}$ $(h \times v, \mathrm{FWHM})$ at the sample position. The obtained extended $x$-ray absorption fine structure (EXAFS) spectra were processed with the ATHENA and ARTEMIS programs included in the IFEFFIT package [49]. The spectra were weighted by $k^{1}, k^{2}, k^{3}$ within a $k$ range of $3.8-13.2 \AA^{-1}$. The data was modeled in the real space with Hanning windows and $d k=2$ within a range of 1.0-2.7 $\AA$ using a shell-by-shell approach. Multiple scattering paths do not contribute in the modeled $R$ region. The single scattering paths were calculated with FEFF6 for the crystal structure of $\alpha-\mathrm{FeSi}_{2}$. The amplitude reduction factor was set to 0.7 and was fixed during the fitting process. It was obtained by modeling the EXAFS spectrum of the $\alpha$-Fe foil used for calibration. The Debye-Waller factors of $\mathrm{Si}$ were variable fit parameters, whereas for $\mathrm{Fe}$ the mean square displacement values obtained from the NIS experiment were used.

The Fe-partial PDOS was obtained [50] by NIS experiments [51,52] performed at the Dynamics Beamline P01 [53] at PETRA III and the Nuclear Resonance Beamline ID18 [54] at the ESRF. At both beamlines the measurements were performed in grazing-incidence geometry with an incidence angle $<0.2^{\circ}$ and an x-ray beam with dimensions of $1.5 \times 0.01 \mathrm{~mm}(h \times v$, FWHM $)$. The energy dependence of the probability for nuclear inelastic absorption was measured by tuning the energy of the $\mathrm{x}$-ray beam around the ${ }^{57} \mathrm{Fe}$ resonance at $14.413 \mathrm{keV}$ with an energy resolution of $0.7 \mathrm{meV}$ for S1, S2 (ID18), $1.0 \mathrm{meV}$ for S3, S4 (P01), and $1.3 \mathrm{meV}$ for S5, S6 (P01). Sample S4 was transported to the beamline P01 


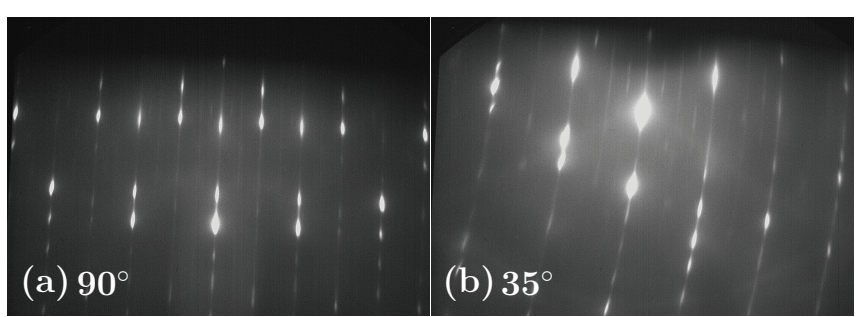

FIG. 1. RHEED patterns of sample $\mathrm{S} 2$ obtained with $E=28 \mathrm{keV}$ (a) perpendicular to the NWs (along $\alpha-\mathrm{FeSi}_{2}[44 \overline{1}]$ ) and (b) at an angle of $35^{\circ}$ between the electron beam and the NWS.

and measured under UHV condition $\left(P<5 \times 10^{-7} \mathrm{~Pa}\right)$ in a dedicated chamber [55].

First-principles calculations were performed within the density functional theory implemented in the VASP code [56,57], employing the generalized gradient approximation $[58,59]$. The phonon dispersions and PDOS were calculated using the direct method [60] incorporated into the PHONON program [61]. To account for the tensile epitaxial strain between the $\mathrm{FeSi}_{2}$ crystal and the $\mathrm{Si}(110)$ surface, the calculations were performed for $0.5 \%$ tensile-strained $\alpha$-phase $\mathrm{FeSi}_{2}(a=2.716 \AA, c=5.166 \AA)$. Further details are given elsewhere [62].

In order to correctly compare the experimental and theoretical PDOS, the variation of the energy resolution in the NIS experiments was accounted for in the following way. The $a b$ initio calculated energy dependence of the probability for nuclear inelastic absorption was convoluted with a Voigt profile, with the FWHM corresponding to the energy resolution used for the respective sample. Subsequently, the PDOS was calculated and used for the fitting process of the respective sample.

\section{RESULTS AND DISCUSSION}

\section{A. Structural investigation}

Figure 1 shows generic RHEED patterns of NWs obtained with the wave vector of the electron beam being oriented (a) $90^{\circ}$ and (b) $35^{\circ}$ with respect to the NWs. At $90^{\circ}$ the pattern is composed of several diffraction spots superimposed on straight streaks. When the angle between the NWs and the wave vector of the electron beam is reduced to $35^{\circ}$, the streaks are bent and the diffraction spots follow their curvature. This observation is explained by the reciprocal space planes of one-dimensional atomic chains with high crystalline order along the chain orientation [63-65] and confirms the formation of single-crystalline, unidirectionally aligned NWs. The RHEED images obtained for S1-S6 revealed the same pattern throughout the entire range of growth parameters, indicating that for all samples the NWs exhibit the same crystal structure. A detailed discussion of the RHEED results is given in the Supplemental Material [66].

In Fig. 2 the experimental EXAFS spectra in $k$ space of samples S7, S2, and S6 are compared to the respective best-fit results. Since S7 was grown at very similar conditions as S1 (see Table I), it is concluded that the NWs of these two samples exhibit the same crystal structure. In Table II the interatomic distances and coordination numbers of the $\mathrm{Si}$ and $\mathrm{Fe}$
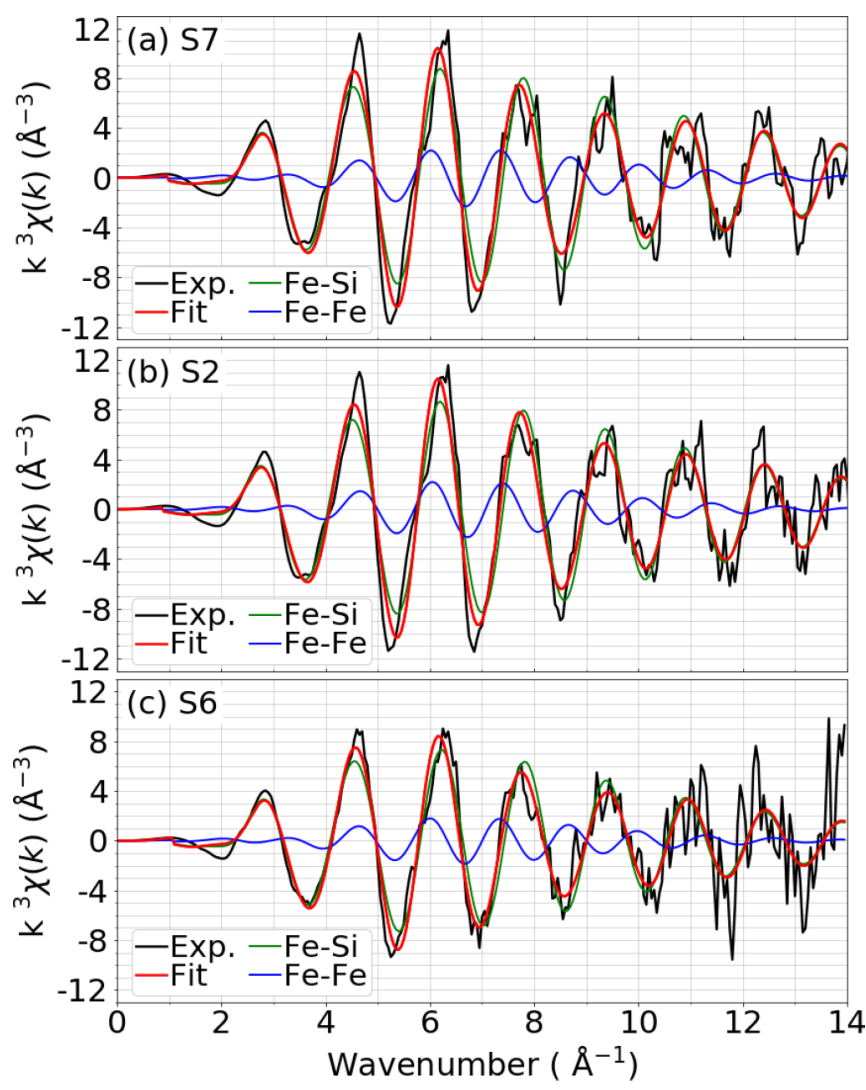

FIG. 2. Fe $K$-edge EXAFS spectra of (a) S7, (b) S2, and (c) S6 compared with the respective best-fit results and Fe-Fe, Fe-Si scattering path subspectra. For the modeling the $\alpha-\mathrm{FeSi}_{2}$ crystal structure was assumed.

nearest neighbors, obtained by modeling of the experimental data, are compared with the values theoretically predicted for the $\mathrm{FeSi}_{2}$ phases formed on Si surfaces. For the determination of the theoretical values it has to be considered that in single crystals the intensity of the EXAFS signal depends on the orientation of the incoming x-ray beam relative to the crystal axes. All spectra were measured with the wave vector of the $\mathrm{x}$-ray beam being parallel to the NWs, i.e., oriented along $\mathrm{Si}[\overline{1} 10]$. The crystal directions being parallel to $\mathrm{Si}[\overline{1} 10]$ are $\alpha-\mathrm{FeSi}_{2}[1 \overline{1} 0], \beta-\mathrm{FeSi}_{2}[010], \gamma-\mathrm{FeSi}_{2}[\overline{1} 10]$, and $s-\mathrm{FeSi}_{2}[\overline{1} 10]$ $[67,68]$. Correspondingly, the coordination numbers given in Table II were calculated with the x-ray beam projected along the respective $\mathrm{FeSi}_{2}$ crystal direction. The results for both parameters exclude the formation of $\beta-, s-$, or $\gamma-\mathrm{FeSi}_{2}$ and, in agreement with an earlier report [10], reveal that the investigated NWs exhibit the tetragonal $\alpha$ phase. Furthermore, the values obtained from the modeling of the EXAFS data show an increase of the Debye-Waller factor of the Si atoms in the smallest wires (S6). The fit results for the interatomic distances do not show a size-dependent behavior, while the coordination numbers for $\mathrm{Fe}-\mathrm{Si}$ and $\mathrm{Fe}-\mathrm{Fe}$ are reduced in $\mathrm{S} 6$ compared to S7 and S2. Most likely the reason for this is the increased interface-to-volume ratio in the smallest NWs of S6 compared to S7 and S2.

For the growth of $\alpha-\mathrm{FeSi}_{2}$ on $\mathrm{Si}(111)[62,67,69]$ and $\mathrm{Si}(001)[70,71]$ the commonly reported epitaxial relation is 
TABLE II. Debye-Waller factor $\left(\sigma^{2}\right)$, coordination numbers, and interatomic distances $(d)$ obtained from modeling of the experimental EXAFS spectra and theoretical values for the expected $\mathrm{FeSi}_{2}$ phases. The coordination numbers were calculated with the x-ray beam projected along the respective crystal direction parallel to $\mathrm{Si}[\overline{1} 10]$, i.e., along $\alpha-\mathrm{FeSi}_{2}[1 \overline{1} 0], \beta-\mathrm{FeSi}_{2}[010], \gamma-\mathrm{FeSi}_{2}[\overline{1} 10]$, and $s$-FeSi ${ }_{2}[110]$. The $\sigma^{2}$ values for the Fe-Si scattering path are derived from the modeling of the experimental EXAFS spectra; for the $\mathrm{Fe}-\mathrm{Fe}$ scattering path they were fixed to the mean-square displacement values obtained from the NIS experiments. The theoretical values for $\alpha$ and $\beta$ phase are obtained from ICSD 5257 and 9119, respectively, and for $s$ and $\gamma$ phase no database values available.

\begin{tabular}{lcccc}
\hline \hline & $\begin{array}{c}\text { Scattering } \\
\text { path }\end{array}$ & $\sigma^{2}\left(10^{-2} \AA^{2}\right)$ & $\begin{array}{c}\text { Coordination } \\
\text { number }\end{array}$ & $d(\AA)$ \\
\hline \multirow{3}{*}{ S7 } & $\mathrm{Fe}-\mathrm{Si}$ & $0.41 \pm 0.05$ & $7.4 \pm 0.4$ & $2.36 \pm 0.01$ \\
& $\mathrm{Fe}-\mathrm{Fe}$ & $1.00 \pm 0.02$ & $2.6 \pm 0.3$ & $2.68 \pm 0.01$ \\
S2 & $\mathrm{Fe}-\mathrm{Si}$ & $0.41 \pm 0.04$ & $7.3 \pm 0.3$ & $2.35 \pm 0.01$ \\
& $\mathrm{Fe}-\mathrm{Fe}$ & $1.01 \pm 0.02$ & $2.8 \pm 0.3$ & $2.67 \pm 0.01$ \\
S6 & $\mathrm{Fe}-\mathrm{Si}$ & $0.52 \pm 0.08$ & $6.6 \pm 0.5$ & $2.35 \pm 0.01$ \\
& $\mathrm{Fe}-\mathrm{Fe}$ & $1.05 \pm 0.02$ & $2.3 \pm 0.5$ & $2.69 \pm 0.01$ \\
$\alpha$-phase & $\mathrm{Fe}-\mathrm{Si}$ & - & 8 & 2.36 \\
& $\mathrm{Fe}-\mathrm{Fe}$ & - & 2 & 2.70 \\
$\beta$-phase & $\mathrm{Fe}-\mathrm{Si}$ & - & 6 & 2.36 \\
& $\mathrm{Fe}-\mathrm{Fe}$ & - & 2 & 2.97 \\
\multirow{2}{*}{-phase } & $\mathrm{Fe}-\mathrm{Si}$ & - & 4 & 2.39 \\
& $\mathrm{Fe}-\mathrm{Fe}$ & - & 4 & 2.76 \\
$\gamma$-phase & $\mathrm{Fe}-\mathrm{Si}$ & - & 4 & 2.33 \\
\hline \hline
\end{tabular}

$\mathrm{Si}\{111\} \| \alpha-\mathrm{FeSi}_{2}\{112\}$. In this configuration, the lattice mismatch is minimized if $\mathrm{Si}\langle\overline{1} 10\rangle \| \alpha-\mathrm{FeSi}_{2}\langle 1 \overline{1} 0\rangle$ [67]. Translated on the $\mathrm{Si}(110)$ surface, this leads to $\mathrm{Si}(111) \| \alpha-\mathrm{FeSi}_{2}(112)$ and $\mathrm{Si}[\overline{1} 10] \| \alpha-\mathrm{FeSi}_{2}[1 \overline{1} 0]$. In Fig. 3 the corresponding orientation of the $\alpha-\mathrm{FeSi}_{2}$ unit cell on the $\mathrm{Si}(110)$ surface is

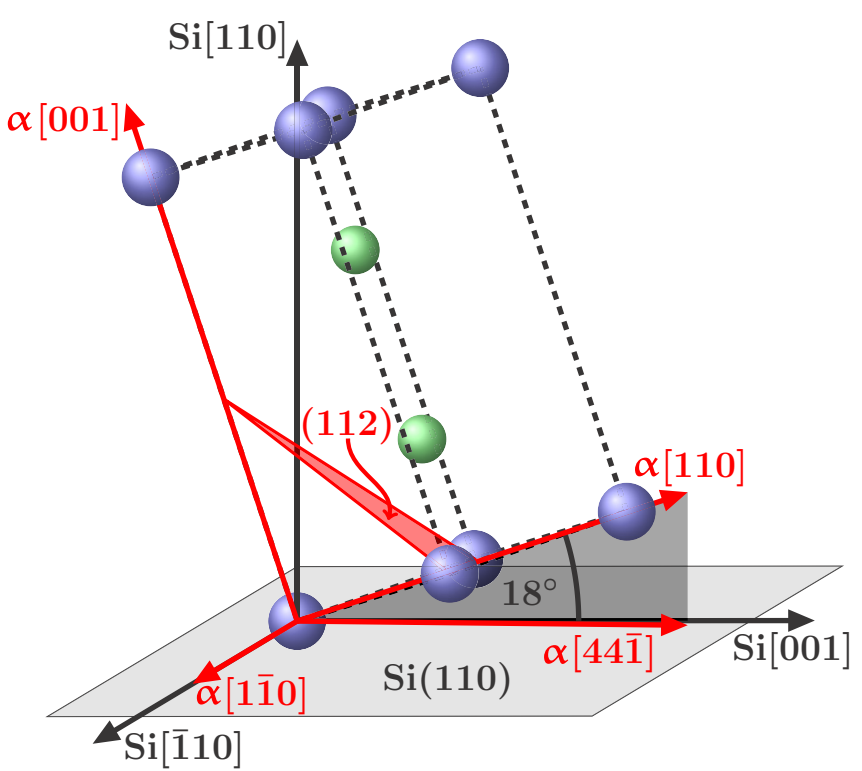

FIG. 3. Orientation of the $\alpha-\mathrm{FeSi}_{2}$ unit cell on the $\mathrm{Si}(110)$ surface. The $\mathrm{Si}\left(\alpha-\mathrm{FeSi}_{2}\right)$ directions/planes are given in black/gray (red/light red). Fe atoms are depicted in blue, $\mathrm{Si}$ atoms in green.

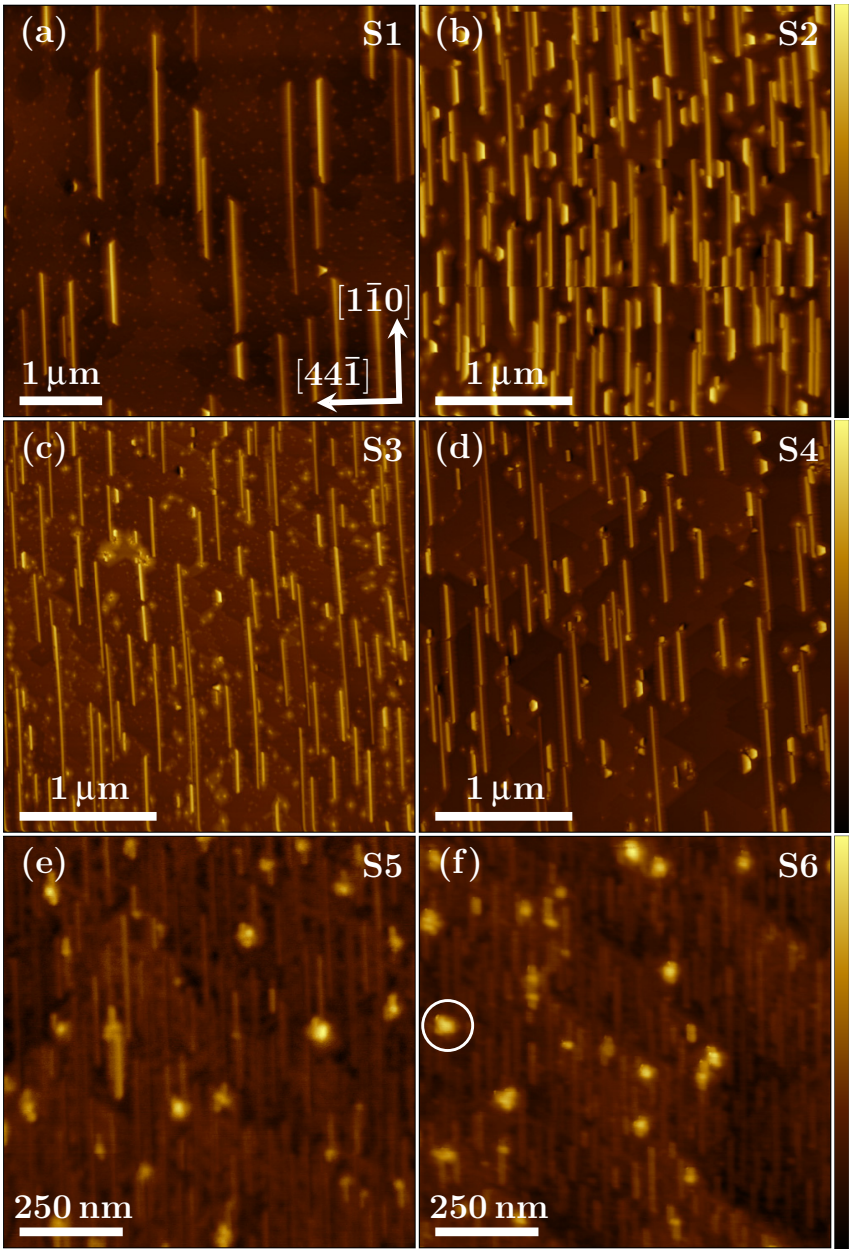

FIG. 4. AFM images of the indicated samples with (a) height scale (hs): 0-59 nm, (b) hs: 0-39 nm, (c) hs: 0-30 nm, (d) hs: 0-37 nm, (e) hs: 0-10 nm, and (f) hs: 0-12 nm. In (a) the crystallographic directions of the $\alpha-\mathrm{FeSi}_{2}$ crystal are indicated. The white circle in (f) marks an exemplary copper contamination.

depicted. The lattice mismatch [defined as $\left.\left(a_{\mathrm{Si}}-a_{\mathrm{FeSi}_{2}}\right) / a_{\mathrm{Si}}\right)$ ] is $0.5 \%$ along $\mathrm{Si}[\overline{1} 10]$ and $1 \%$ along $\mathrm{Si}[001]$, and the tilt angle between $\alpha-\mathrm{FeSi}_{2}[110]$ and $\mathrm{Si}[001]$ amounts to $18^{\circ}$. Furthermore, this configuration implies that $\alpha-\mathrm{FeSi}_{2}[44 \overline{1}]$ is $0.5^{\circ}$ off $\mathrm{Si}[001]$.

Figure 4 shows an overview of the AFM images of S1S6. For all samples the NWs are unidirectionally aligned along $\mathrm{Si}[\overline{1} 10]$, as reported for the growth of $\mathrm{FeSi}_{2}$ on $\mathrm{Si}(110)[5,7,9,10]$. The epitaxial relation discussed above implies that the NWs are formed along $\mathrm{Si}[\overline{1} 10] \| \alpha-\mathrm{FeSi}_{2}[1 \overline{1} 0]$. Furthermore, due to the small deviation of $0.5^{\circ}$ we approximate that $\mathrm{Si}[001] \| \alpha-\mathrm{FeSi}_{2}[44 \overline{1}]$ [Fig. 4(a)]. The average width $\bar{w}$ of the NWs, calculated from AFM line scans [66], are given in Table I. As expected, an increase of the growth temperature $T_{G}$ or the amount of deposited iron $\theta_{F e}$ leads to NWs with larger dimensions. The AFM images of S5 and S6 exhibit additional round islands, an example of which is marked in Fig. 4(f). These structures occur after the removal of the native $\mathrm{SiO}_{2}$ layer and by $\mathrm{x}$-ray photoelectron spectroscopy were identified as a copper contamination. Since the NIS technique is sensitive solely to the ${ }^{57} \mathrm{Fe}$ nuclei $[51,52,72]$, the $\mathrm{Cu}$ islands do not contribute to the obtained PDOS of the NWs. 


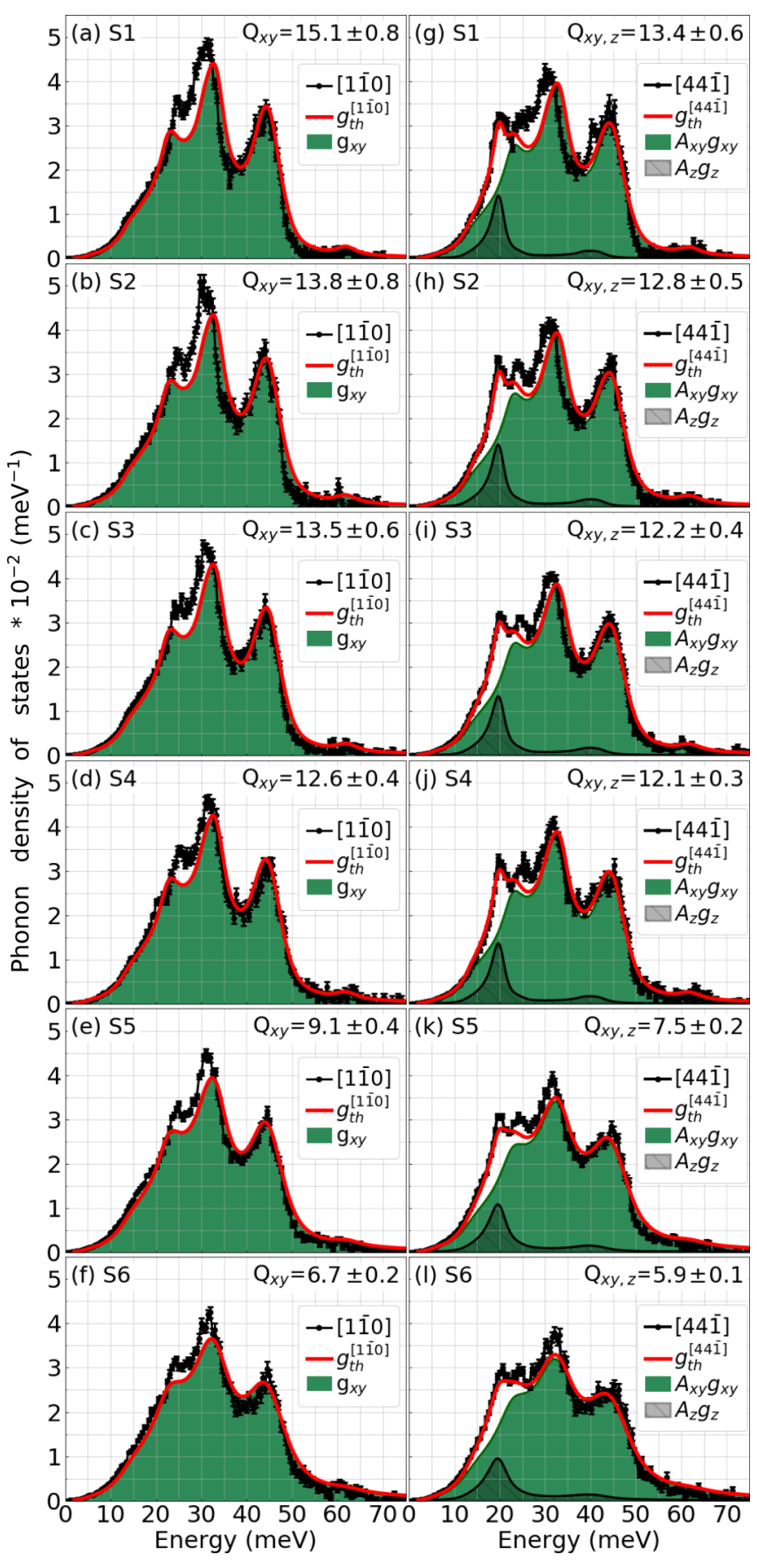

FIG. 5. Fe-partial PDOS of the indicated samples measured (a)(f) along $\alpha-\mathrm{FeSi}_{2}[1 \overline{1} 0]$ and (g)-(l) along $\alpha-\mathrm{FeSi}_{2}[44 \overline{1}]$. The given error bars represent the $1-\sigma$ uncertainty. The experimental data (symbols) is compared with the results of the least-squares fit (solid red line), decomposed into its weighted $x y\left(A_{x y} g_{x y}\right)$ and $z\left(A_{z} g_{z}\right)$ contributions (for details see text). The resulting quality factors $Q_{x y}$ and $Q_{x y, z}$ of the DHO function are also given.

\section{B. Lattice dynamics}

In Fig. 5 the Fe-partial PDOS of S1-S6 obtained with the wave vector of the $\mathrm{x}$-ray beam being parallel to $\alpha-\mathrm{FeSi}_{2}[1 \overline{1} 0]$ (left column) and $\alpha-\mathrm{FeSi}_{2}[44 \overline{1}]$ (right column) are depicted. A comparison of the PDOS along and across the NWs shows a vibrational anisotropy with pronounced differences around
$20 \mathrm{meV}$. Furthermore, the reduction of the average NW width $\bar{w}$ from 24 (S1) to $3 \mathrm{~nm}$ (S6) leads to a broadening of the peaks.

Previous $a b$ initio calculations of the direction-projected Fe-partial PDOS of the tetragonal $\alpha-\mathrm{FeSi}_{2}$ showed a decoupling of vibrations with $x y$ and $z$ polarization [62]. The Fe-partial PDOS of the $x y$-polarized vibrations consists of peaks at 24, 33, and $45 \mathrm{meV}$, while the z-polarized vibrations are mostly localized at $20 \mathrm{meV}$ with a minor plateau at around $40 \mathrm{meV}$. The experimental PDOS obtained with the wave vector of the X-ray beam being parallel to a certain crystallographic direction of the NWs is composed of a specific combination of $x y$ - and $z$-polarized phonons $[62,73,74]$. The relative contributions of $x y-\left(A_{x y}\right)$ and $z-\left(A_{z}\right)$ polarized phonons can be calculated [66] considering the orientation of the $\alpha-\mathrm{FeSi}_{2}$ unit cell and amount to $A_{x y}^{[1 \overline{1} 0]}=1$ and $A_{z}^{[1 \overline{1} 0]}=0$ for $\alpha-\mathrm{FeSi}_{2}[1 \overline{1} 0]$ and $A_{x y}^{[44 \overline{1}]}=0.9$ and $A_{z}^{[44 \overline{1}]}=0.1$ for $\alpha-\mathrm{FeSi}_{2}[44 \overline{1}]$. Consequently, the observed vibrational anisotropy originates from the specific orientation of the $\alpha-\mathrm{FeSi}_{2}$ unit cell on the $\mathrm{Si}(110)$ surface (see Fig. 3).

The observed damping of the PDOS features upon reduction of the NW size can be quantified by comparison of the experimental results with the $a b$ initio calculations [62]. The damping originates from phonon scattering at defects at interfaces and surfaces, as well as within the crystal [75], and can be described by the damped harmonic oscillator (DHO) function [76]. The DHO function introduces an energy-dependent broadening of the spectral features quantified by the quality factor $Q$, which is inversely proportional to the strength of the damping. The experimental PDOS data were modeled by convolution of the $a b$ initio calculated PDOS, obtained for a $0.5 \%$ tensile strained $\alpha-\mathrm{FeSi}_{2}$ crystal, with the DHO function. The strength of the damping in the respective sample is quantified by the $Q$ values obtained using the least-squares method.

For measurements along $\alpha-\mathrm{FeSi}_{2}[1 \overline{1} 0]$ (along the NWs), the PDOS consists of $x y$-polarized vibrations only and the experimental data can be described by

$$
g_{t h}^{[1 \overline{1} 0]}=g_{x y}\left(E, Q_{x y}\right),
$$

with $g_{x y}$ being the $a b$ initio calculated $x y$-polarized Fe-partial PDOS convoluted with the DHO function with a quality factor $Q_{x y}$. In Figs. 5(a)-5(f) $g_{t h}^{[1 \overline{1} 0]}$ is compared with the respective experimental PDOS. In general, a very good agreement is observed between experiment and theory. While the peak at around $45 \mathrm{meV}$ occurs at the same positions in the experimentally determined and $a b$ initio calculated PDOS, the minor peak at around $25 \mathrm{meV}$ is shifted by $1.5 \mathrm{meV}$ to lower energy, and the peak at around $33 \mathrm{meV}$ is shifted by about $1 \mathrm{meV}$ to higher energy in the ab initio calculated PDOS. Most likely these differences occur due to a more complicated strain distribution in the $\alpha-\mathrm{FeSi}_{2}$ crystal than the assumed isotropic $0.5 \%$ tensile strain.

The PDOS obtained along $\alpha-\mathrm{FeSi}_{2}[44 \overline{1}]$ (across the NWs) is modeled by the weighted sum of the $a b$ initio calculated $x y$ - and $z$-polarized PDOS, convoluted with the DHO function with a quality factor $Q_{x y, z}$ :

$$
g_{t h}^{[44 \overline{1}]}=A_{x y}^{[44 \overline{1}]} g_{x y}\left(E, Q_{x y, z}\right)+A_{z}^{[44 \overline{1}]} g_{z}\left(E, Q_{x y, z}\right) .
$$




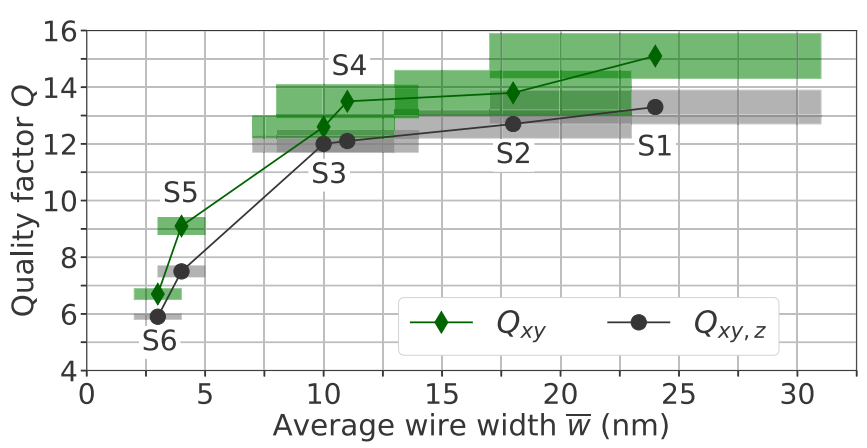

FIG. 6. Quality factors $Q_{x y}$ and $Q_{x y, z}$ (Fig. 5) as a function of average NW width $\bar{w}$ (Table I). The shaded boxes denote the uncertainties in $Q_{x y}, Q_{x y, z}$, and $\bar{w}$.

In Figs. $5(\mathrm{~g})-5(1) g_{t h}^{[44 \overline{1}]}$ is plotted with the respective $x y$ $\left(A_{x y} g_{x y}\right)$ and $z\left(A_{z} g_{z}\right)$ contributions and the corresponding $Q_{x y, z}$ values obtained from the fit [77]. A very good agreement between experiment and theory is observed. The peak of the $z$-polarized phonons at around $20 \mathrm{meV}$ occurs at the same energies in theory and experiment. The $x y$-polarized vibrations along $\alpha-\mathrm{FeSi}_{2}[44 \overline{1}]$ are also well reproduced by the model, except for small shifts of the peaks at 25 and $33 \mathrm{meV}$, which are also observed in the measurements along $\alpha-\mathrm{FeSi}_{2}[1 \overline{1} 0]$ and are attributed to a complex strain distribution. On average, the $Q_{x y, z}$ values obtained for the PDOS across the NWs are reduced by $10 \%$ compared to the $Q_{x y}$ values obtained for the PDOS along the NWs. The reason for the slightly stronger damping of the phonons propagating across the NWs could be the smaller size of the $\alpha-\mathrm{FeSi}_{2}$ crystal along this direction.

In Fig. 6 the quality factors obtained from the least-squares fits for S1-S6 along $\alpha-\mathrm{FeSi}_{2}[1 \overline{1} 0]$ and $\alpha-\mathrm{FeSi}_{2}$ [441̄] are depicted as a function of $\bar{w}$. The $Q_{x y}$ and $Q_{x y, z}$ values of S1-S4 show a slight decrease in the range of $24 \mathrm{~nm} \geqslant \bar{w} \geqslant 10 \mathrm{~nm}$, whereas upon reduction of $\bar{w}$ below $10 \mathrm{~nm}$ in S5 and S6, $Q_{x y}$ and $Q_{x y, z}$ are significantly reduced. To comprehend this trend, the interface-to-volume ratio of the NWs has to be considered. In the volume part, i.e., the core of the NWs, the atoms are located in a bulklike environment with a high degree of crystalline order. At the interface towards the substrate the defect density is generally increased and thus the scattering of phonons is enhanced. For the smallest wires of S5 and S6, the interface-to-volume ratio is significantly higher, consequently, $Q_{x y}$ and $Q_{x y, z}$ are distinctly reduced compared to S1-S4.

A study of the lattice dynamics of epitaxial $\alpha-\mathrm{FeSi}_{2}$ nanoislands on $\mathrm{Si}(111)$ unveiled a polarization dependence of the phonon damping, i.e., a stronger damping of $z$-polarized phonons, in islands with average heights below $10 \mathrm{~nm}$ [62]. To examine if this effect is also present in the investigated NWs, the PDOS obtained along $\alpha-\mathrm{FeSi}_{2}[44 \overline{1}]$ were fitted by the weighted sum of the $a b$ initio calculated $x y$ - and $z$-polarized PDOS convoluted with DHO functions with independent quality factors. Although the results indicate that this effect might also be present in S5 and S6, the low intensity of the peak at $20 \mathrm{meV}$ does not allow for a definite conclusion.

In Fig. 7 the Fe-partial reduced PDOS $\left[g(E) / E^{2}\right]$ of S1-S6 is shown. Along both directions no systematic increase in lowenergy states is observed with reduction of $\bar{w}$ from $24 \mathrm{~nm}(\mathrm{~S} 1)$

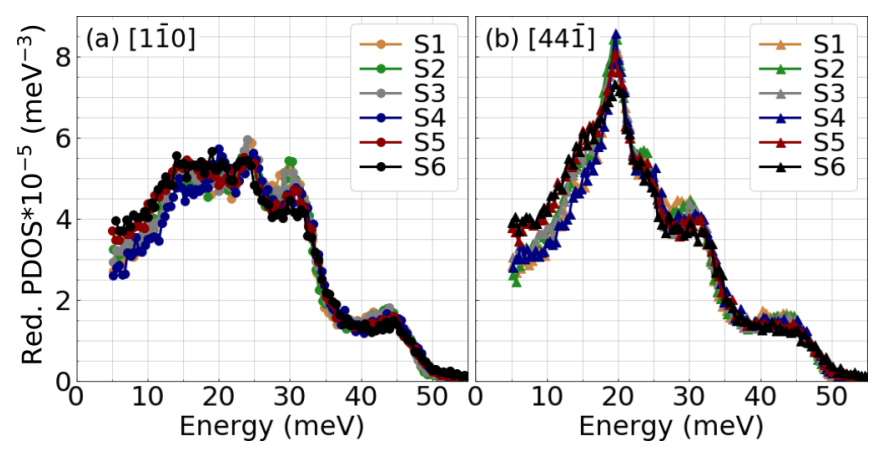

FIG. 7. Fe-partial reduced PDOS $\left[g(E) / E^{2}\right]$ of the indicated samples obtained (a) along $\alpha-\mathrm{FeSi}_{2}[1 \overline{1} 0]$ and (b) along $\alpha-\mathrm{FeSi}_{2}[44 \overline{1}]$.

to $10 \mathrm{~nm}(\mathrm{~S} 3)$. However, the samples with the smallest NWs, S5 and S6, show an increase of states in the region from 5 to $15 \mathrm{meV}$. Such an enhancement of low-energy states has been observed in the PDOS of thin films [78-80] and surfaces [81]. It is attributed to interface/surface-specific vibrational modes, which are more pronounced in the smallest NWs.

A comparison of the PDOS and reduced PDOS of the capped and uncapped NWs of S3 and S4 unveils only minor deviations (see Fig. 5 in Supplemental Material [66]). The negligible influence of the capping layer can be explained by the endotaxial growth mechanism of the NWs, which results in a large $\mathrm{NW} /$ substrate interface and a small fraction of atoms located at the surface of the NWs.

\section{Thermodynamic and elastic properties}

The thermodynamic and elastic properties obtained from the $a b$ initio calculated and experimentally determined PDOS [72] are given in Table III. The experiments show an average decrease of the mean force constant $F$ by $1.6 \%$ along [44ī] compared to [1 10$]$, while the mean-square displacement $\left\langle x^{2}\right\rangle$ and the vibrational entropy $S_{V}$ on average increase by $5 \%$ and $2 \%$, respectively. These differences originate from the vibrational anisotropy of the tetragonal $\alpha-\mathrm{FeSi}_{2}$ unit cell. The contribution of $z$-polarized phonons along $\alpha$-FeSi 2 [44i ] induces the observed softening of the crystal compared to $\alpha-\mathrm{FeSi}_{2}[1 \overline{1} 0]$.

The reduction of $\bar{w}$ of the NWs from S1-S6 leads to an

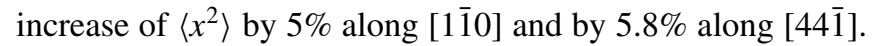
This is in agreement with the trend observed in the $\sigma^{2}$ values obtained for the Fe-Si scattering path by modeling the EXAFS data (see Table II). $F$ is also slightly increased from S1 to S6, most likely due to the enhancement of high-energy states above the cutoff energy, induced by the broadening of the peak at $45 \mathrm{meV}$. A similar behavior was observed in $\alpha-\mathrm{FeSi}_{2}$ nanoislands [62]. A comparison with the theoretically expected values for bulk $\alpha-\mathrm{FeSi}_{2}$ shows that the $\left\langle x^{2}\right\rangle$ and $S_{V}$ values are on average increased by $8 \%$ and $2 \%$, respectively, in the smallest NWs. The values of the heat capacity $C_{V}$ coincide within the uncertainty for S1-S6 in both directions.

The low-energy part of the PDOS in all samples can be described by the Debye model: $g(E)=\alpha E^{2}$. The coefficient $\alpha$ is on average increasing as the NW size is decreasing (Fig. 7). Using $\alpha$, the sound velocity $v_{S}$ in $\mathrm{S} 1-\mathrm{S} 6$ was calculated [82]. 
TABLE III. Fe-partial mean force constant $F$, mean-square displacement $\left\langle x^{2}\right\rangle$, vibrational entropy $S_{V}$, and heat capacity $C_{V}$ calculated from the $a b$ initio and the experimental PDOS of S1-S6 projected along $\alpha-\mathrm{FeSi}_{2}[1 \overline{1} 0]$ and $\alpha-\mathrm{FeSi}_{2}[44 \overline{1}]$. The coefficient $\alpha$ derived from the low-energy part of the reduced PDOS $\left[g(E) / E^{2}=\alpha\right]$ and the sound velocity $v_{S}$ are also given.

\begin{tabular}{|c|c|c|c|c|c|c|c|}
\hline & Direction & $F(\mathrm{~N} / \mathrm{m})$ & $\left\langle x^{2}\right\rangle\left(\AA^{2}\right)$ & $S_{V}\left(k_{B} /\right.$ atom $)$ & $C_{V}\left(k_{B} /\right.$ atom $)$ & $\alpha\left(10^{-5} \mathrm{meV}^{-3}\right)$ & $v_{S}(\mathrm{~m} / \mathrm{s})$ \\
\hline \multirow[t]{2}{*}{ Theory } & {$[1 \overline{1} 0]$} & $254 \pm 5$ & $0.0096 \pm 0.0002$ & $2.62 \pm 0.02$ & $2.60 \pm 0.02$ & - & 4988 \\
\hline & {$[44 \overline{1}]$} & $245 \pm 5$ & $0.0096 \pm 0.0002$ & $2.68 \pm 0.02$ & $2.61 \pm 0.02$ & - & - \\
\hline S1 & {$[44 \overline{1}]$} & $245 \pm 5$ & $0.0104 \pm 0.0002$ & $2.74 \pm 0.02$ & $2.62 \pm 0.02$ & $3.03 \pm 0.07$ & $4780 \pm 100$ \\
\hline \multirow[t]{2}{*}{ S2 } & {$[1 \overline{1} 0]$} & $250 \pm 5$ & $0.0101 \pm 0.0002$ & $2.67 \pm 0.02$ & $2.60 \pm 0.02$ & $3.51 \pm 0.07$ & $4550 \pm 100$ \\
\hline & {$[44 \overline{1}]$} & $245 \pm 5$ & $0.0105 \pm 0.0002$ & $2.73 \pm 0.02$ & $2.61 \pm 0.02$ & $3.24 \pm 0.08$ & $4670 \pm 120$ \\
\hline S3 & {$[44 \overline{1}]$} & $247 \pm 5$ & $0.0105 \pm 0.0002$ & $2.72 \pm 0.02$ & $2.61 \pm 0.02$ & $3.37 \pm 0.07$ & $4610 \pm 100$ \\
\hline \multirow[t]{2}{*}{ S4 } & {$[1 \overline{1} 0]$} & $256 \pm 5$ & $0.0096 \pm 0.0002$ & $2.64 \pm 0.02$ & $2.59 \pm 0.02$ & $3.01 \pm 0.09$ & $4780 \pm 140$ \\
\hline & {$[44 \overline{1}]$} & $251 \pm 5$ & $0.0102 \pm 0.0002$ & $2.69 \pm 0.02$ & $2.59 \pm 0.02$ & $3.13 \pm 0.04$ & $4730 \pm 60$ \\
\hline \multirow[t]{2}{*}{ S5 } & {$[1 \overline{1} 0]$} & $259 \pm 5$ & $0.0104 \pm 0.0002$ & $2.66 \pm 0.02$ & $2.59 \pm 0.02$ & $3.69 \pm 0.04$ & $4480 \pm 50$ \\
\hline & {$[44 \overline{1}]$} & $250 \pm 5$ & $0.0110 \pm 0.0002$ & $2.74 \pm 0.02$ & $2.60 \pm 0.02$ & $3.82 \pm 0.05$ & $4420 \pm 60$ \\
\hline S6 & {$[1 \overline{1} 0]$} & $255 \pm 5$ & $0.0105 \pm 0.0002$ & $2.68 \pm 0.02$ & $2.59 \pm 0.02$ & $3.96 \pm 0.05$ & $4370 \pm 60$ \\
\hline
\end{tabular}

For comparison the theoretical value for [11 10$]$, determined from the slopes of the three acoustic branches calculated along $\Gamma-\mathrm{M}$ direction, is also given. The experimental values are clearly reduced compared to the theoretical value. The reason for these differences is that a perfect crystal is assumed for the ab initio calculations, whereas in the NWs the propagation of sound waves is decelerated by scattering at defects, which are mostly present at interfaces. Since the interface-to-volume ratio is increased when the NWs dimensions are reduced, $v_{S}$ is also reduced from $\mathrm{S} 1-\mathrm{S} 6$ by $9 \%$.

\section{CONCLUSIONS}

Endotaxial $\mathrm{FeSi}_{2} \mathrm{NWs}$ were grown on $\mathrm{Si}(110)$ by reactive deposition epitaxy. Systematic RHEED and AFM studies unveiled the formation of single-crystalline, unidirectionally aligned NWs with average widths $\bar{w}$ from 24 to $3 \mathrm{~nm}$ and lengths from several micrometers to about $100 \mathrm{~nm}$. A combined experimental and theoretical EXAFS study demonstrated that the NWs exhibit the metastable, surface-stabilized $\alpha-\mathrm{FeSi}_{2}$ crystal structure.

The Fe-partial PDOS was determined along and across the NWs by NIS experiments performed at room temperature. A pronounced vibrational anisotropy originating from the specific orientation of the tetragonal $\alpha-\mathrm{FeSi}_{2}$ unit cell on the $\mathrm{Si}(110)$ surface was unveiled. Modeling of the experimental data with first-principles calculations showed that upon reduction of $\bar{w}$ from 24 to $3 \mathrm{~nm}$, the features of the PDOS broaden significantly. This is attributed to phonon scattering at the NW/substrate interface, which is particularly strong in the smallest NWs characterized with the highest interface-to- volume ratio. Furthermore, the reduction of $\bar{w}$ from 24 to $3 \mathrm{~nm}$ leads to an increase of the mean-square displacement by $5 \%$ and a reduction of the sound velocity by $9 \%$. The damping of lattice vibrations is slightly stronger across the NWs due to the smaller size of the $\alpha-\mathrm{FeSi}_{2}$ crystal along this direction. A comparison of the PDOS of NWs with identical sizes measured with and without a capping layer demonstrates that the influence of surface-specific vibrational modes is negligible due to the endotaxial growth mechanism of the NWs.

The presented results on the lattice dynamics and thermoelastic properties of $\mathrm{FeSi}_{2}$ nanowires are expected to be generally valid for the technologically important class of endotaxial silicide nanowires.

\section{ACKNOWLEDGMENTS}

S.S. acknowledges financial support from the Helmholtz Association (Grant No. VH-NG-625) and BMBF (Grant No. 05K16VK4). P.P. acknowledges support by the Narodowe Centrum Nauki (NCN, National Science Centre) under Project No. 2017/25/B/ST3/02586 and the access to ESRF financed by the Polish Ministry of Science and High Education, Decision No. DIR/WK/2016/19. The European Synchrotron Radiation Facility is acknowledged for beamtime provision at the Nuclear Resonance Beamline ID18. We thank Mr. J.-P. Celse for technical assistance during the experiment at ID18. We acknowledge DESY (Hamburg, Germany), a member of the Helmholtz Association HGF, for the provision of experimental facilities. Parts of this research were carried out at the High Resolution Dynamics Beamline P01 at PETRA III. We thank Mr. C. Hagemeister and Mr. F.-U. Dill for technical assistance during the experiment at P01.
[1] S. P. Murarka, Intermetallics 3, 173 (1995).

[2] L. J. Chen, Silicide Technology for Integrated Circuits (Institution of Electrical Engineers, London, 2004).
[3] L. J. Chen, JOM 57, 24 (2005).

[4] P. A. Bennett, Z. He, D. J. Smith, and F. M. Ross, Thin Solid Films 519, 8434 (2011). 
[5] Z. He, D. J. Smith, and P. A. Bennett, Phys. Rev. Lett. 93, 256102 (2004).

[6] J. C. Mahato, D. Das, N. Banu, B. Satpati, and B. N. Dev, Nanotechnology 28, 425603 (2017).

[7] S. Liang, R. Islam, D. J. Smith, P. A. Bennett, J. R. O’Brien, and B. Taylor, Appl. Phys. Lett. 88, 113111 (2006).

[8] S. Liang, R. Islam, D. J. Smith, and P. A. Bennett, J. Cryst. Growth 295, 166 (2006).

[9] D. Das, J. C. Mahato, B. Bisi, B. Satpati, and B. N. Dev, Appl. Phys. Lett. 105, 191606 (2014).

[10] Z.-Q. Zou, X. Li, X.-Y. Liu, K.-J. Shi, and X.-Q. Guo, Appl. Surf. Sci. 399, 200 (2017).

[11] D. Bozyigit, N. Yazdani, M. Yarema, O. Yarema, W. M. M. Li, S. Volk, K. Vuttivorakulchai, M. Luisier, F. Juranyi, and V. Wood, Nature (London) 531, 618 (2016).

[12] S. P. Hepplestone and G. P. Srivastava, Appl. Phys. Lett. 87, 231906 (2005).

[13] W. Steinhögl, G. Schindler, G. Steinlesberger, and M. Engelhardt, Phys. Rev. B 66, 075414 (2002).

[14] D. Rideau, W. Zhang, Y. M. Niquet, C. Delerue, C. Tavernier, and H. Jaouen, 2011 International Conference on Simulation of Semiconductor Processes and Devices (IEEE, New York, 2011), p. 47.

[15] S. K. Tobler, and P. A. Bennett, J. Appl. Phys. 118, 125305 (2015).

[16] N. Nishiguchi, Y. Ando, and M. N. Wybourne, J. Phys.: Condens. Matter 9, 5751 (1997).

[17] O. M. Mukdadi, S. K. Datta, and M. L. Dunn, J. Appl. Phys. 97, 074313 (2005).

[18] S. P. Hepplestone and G. P. Srivastava, Nanotechnology 17, 3288 (2006).

[19] P. B. Allen, Nano Lett. 7, 11 (2007).

[20] S. Mizuno and N. Nishiguchi, J. Phys.: Condens. Matter 21, 195303 (2009).

[21] Y. Sęngün and S. Durukanoğlu, Phys. Rev. B 83, 113409 (2011).

[22] D. Martínez-Gutiérrez and V. R. Velasco, Surf. Sci. 605, 24 (2011).

[23] S. Mizuno, Jpn. J. Appl. Phys. 53, 07KB02 (2014).

[24] L. Saviot, Phys. Rev. B 97, 155420 (2018).

[25] J. Zou and A. Balandin, J. Appl. Phys. 89, 2932 (2001).

[26] B. A. Glavin, Phys. Rev. Lett. 86, 4318 (2001).

[27] X. Lü and J. H. Chu, J. Appl. Phys., 100, 014305 (2006).

[28] B. Qiu, L. Sun, and X. Ruan, Phys. Rev. B 83, 035312 (2011).

[29] H. Karamitaheri, N. Neophytou, and H. Kosina, J. Appl. Phys. 115, 024302 (2014).

[30] Y. Zhou, X. Zhang, and M. Hu, Nano Lett. 17, 1269 (2017).

[31] Z. Rashid, L. Zhu, and Wu Li, Phys. Rev. B 97, 075441 (2018).

[32] N. Nishiguchi, Physica E 13, 1 (2002).

[33] S. Uno, J. Hattori, K. Nakazato, and M. Nobuya, J. Comput. Electron. 10, 104 (2011).

[34] Y. Yamada, H. Tsuchiya, and M. Ogawa, J. Appl. Phys. 111, 063720 (2012).

[35] I. M. Tienda-Luna, F. G. Ruiz, A. Godoy, L. Donetti, C. Martínez-Blanque, and F. Gámiz, Appl. Phys. Lett. 103, 163107 (2013).

[36] A. Malhotra and M. Maldovan, Nanotechnology 30, 372002 (2019).

[37] B. Li, D. Yu, and S.-L. Zhang, Phys. Rev. B 59, 1645 (1999).
[38] R. P. Wang, G. W. Zhou, Y. L. Liu, S. H. Pan, H. Z. Zhang, D. P. Yu, and Z. Zhang, Phys. Rev. B 61, 16827 (2000).

[39] S. Piscanec, M. Cantoro, A. C. Ferrari, J. A. Zapien, Y. Lifshitz, S. T. Lee, S. Hofmann, and J. Robertson, Phys. Rev. B 68, 241312(R) (2003).

[40] K. W. Adu, H. R. Gutierrez, U. J. Kim, G. U. Sumanasekera, and P. C. Eklund, Nano Lett. 5, 409 (2005).

[41] A. Patsha and S. Dhara, Nano Lett. 18, 7181 (2018).

[42] M. De Luca, C. Fasolato, M. A. Verheijen, Y. Ren, M. Y. Swinkels, S. Kölling, E. P. A. M. Bakkers, R. Rurali, X. Cartoixa, and I. Zardo, Nano Lett. 19, 4702 (2019).

[43] P.-A. Mante, L. Belliard, and B. Perrin, Nanophotonics 7, 1759 (2018).

[44] A. O. Mariager, D. Khakhulin, H. T. Lemke, K. S. Kjaer, L. Guerin, L. Nuccio, C. B. Sørensen, M. N. Nielsen, and R. Feidenhans'l, Nano Lett. 10, 2461 (2010).

[45] F. Kargar, B. Debnath, J.-P. Kakko, A. Säynätjoki, H. Lipsanen, D. L. Nika, R. K. Lake, and A. A. Balandin, Nat. Commun. 7, 13400 (2016).

[46] D. Bessas, W. Töllner, Z. Aabdin, N. Peranio, I. Sergueev, H.-C. Wille, O. Eibl, K. Nielsch, and R. P. Hermann, Nanoscale 5, 10629 (2013).

[47] D. P. Lozano, S. Couet, C. Petermann, G. Hamoir, J. K. Jochum, T. Picot, E. Menendez, K. Houben, V. Joly, V. A. Antohe, M. Y. Hu, B. M. Leu, A. Alatas, A. H. Said, S. Roelants, B. Partoens, M. V. Milosevic, F. M. Peeters, L. Piraux, J. Van de Vondel, et al., Phys. Rev. B 99, 064512 (2019).

[48] B. Krause, S. Darma, M. Kaufholz, H.-H. Gräfe, S. Ulrich, M. Mantilla, R. Weigel, S. Rembold, and T. Baumbach, J. Synchrotron Radiat. 19, 216 (2012).

[49] B. Ravel and M. Newville, J. Synchrotron Radiat. 12, 537 (2005).

[50] V. G. Kohn and A. I. Chumakov, Hyperfine Interact. 125, 205 (2000).

[51] M. Seto, Y. Yoda, S. Kikuta, X. W. Zhang, and M. Ando, Phys. Rev. Lett. 74, 3828 (1995).

[52] W. Sturhahn, T. S. Toellner, E. E. Alp, X. Zhang, M. Ando, Y. Yoda, S. Kikuta, M. Seto, C. W. Kimball, and B. Dabrowski, Phys. Rev. Lett. 74, 3832 (1995).

[53] H.-C. Wille, H. Franz, R. Röhlsberger, W. A. Caliebe, and F. U. Dill, J. Phys.: Conf. Series 217, 0120081 (2010).

[54] R. Rüffer and A. I. Chumakov, Hyperfine Interact. 97, 589 (1996).

[55] S. Ibrahimkutty, A. Seiler, T. Prüßmann, T. Vitova, R. Pradip, O. Bauder, P. Wochner, A. Plech, T. Baumbach, and S. Stankov, J. Synchrotron Radiat. 22, 91 (2015).

[56] G. Kresse and J. Furthmüller, Phys. Rev. B 54, 11169 (1996).

[57] G. Kresse and J. Furthmüller, Comput. Mater. Sci. 6, 15 (1996).

[58] J. P. Perdew, K. Burke, and M. Ernzerhof, Phys. Rev. Lett. 77, 3865 (1996).

[59] J. P. Perdew, K. Burke, and M. Ernzerhof, Phys. Rev. Lett. 78, 1396(E) (1997).

[60] K. Parlinski, Z. Q. Li, and Y. Kawazoe, Phys. Rev. Lett. 78, 4063 (1997).

[61] K. Parlinski, Software PHONON ver. 6.15, Cracow, Poland, 2015.

[62] J. Kalt, M. Sternik, B. Krause, I. Sergueev, M. Mikolasek, D. Bessas, O. Sikora, T. Vitova, J. Göttlicher, R. Steininger, P. T. Jochym, A. Ptok, O. Leupold, H.-C. Wille, A.I. Chumakov, P. 
Piekarz, K. Parlinski, T. Baumbach, and S. Stankov, Phys. Rev. B 101, 165406 (2020).

[63] P. Delescluse and A. Masson, Surf. Sci. 100, 423 (1980).

[64] P. J. Dobson, J. H. Neave, and B. A. Joyce, Surf. Sci. 119, L339 (1982).

[65] G. Wang, S. K. Lok, S. K. Chan, C. Wang, G. K. L. Wong, and I. K. Sou, Nanotechnology 20, 215607 (2009).

[66] See Supplemental Material at http://link.aps.org/supplemental/ 10.1103/PhysRevB.102.195414 for RHEED, EXAFS, and AFM data evaluation, calculation of the direction-projected phonon density of states, and comparison of ex situ and in situ PDOS.

[67] I. Berbezier, J. Chevrier, and J. Derrien, Surf. Sci. 315, 27 (1994).

[68] H. von Känel, N. Onda, H. Sirringhaus, E. Müller-Gubler, S. Concalves-Conto, and C. Schwarz, Appl. Surf. Sci. 70/71, 559 (1993).

[69] K. Kataoka, K. Hattori, Y. Miyatake, and H. Daimon, Phys. Rev. B 74, 155406 (2006).

[70] S. Y. Chen, H. C. Chen, and L. J. Chen, Appl. Phys. Lett. 88, 193114 (2006).

[71] J. H. Won, K. Sato, M. Ishimaru, and Y. Hirotsu, J. Appl. Phys. 100, 014307 (2006).

[72] A. I. Chumakov and W. Sturhahn, Hyperfine Interact. 123, 781 (1999).

[73] A. I. Chumakov, R. Rüffer, A. Q. R. Baron, H. Grünsteudel, H. F. Grünsteudel, and V. G. Kohn, Phys. Rev. B 56, 10758 (1997).
[74] V. G. Kohn, A. I. Chumakov, and R. Rüffer, Phys. Rev. B 58, 8437 (1998).

[75] B. Fultz, Prog. Mater. Sci. 55, 247 (2010).

[76] B. Fåk and B. Dorner, Institute Laue Langevin Technical Report No. 92FA008S, 1992; B. Fåk and B. Dorner, Physica B 234, 1107 (1997).

[77] The experimental data was additionally modeled with $A_{x y}$ and $A_{z}$ being free parameters in the least-squares optimization. The quality factors obtained with this approach coincide within the uncertainty with the values given in Fig. 5, and $A_{x y}$ and $A_{z}$ deviate at most by $2 \%$ from the calculated values of $A_{x y}=0.9$ and $A_{z}=0.1$.

[78] R. Pradip, P. Piekarz, D. G. Merkel, J. Kalt, O. Waller, A. I. Chumakov, R. Rüffer, A. M. Oleś, K. Parlinski, T. Baumbach, and S. Stankov, Nanoscale 11, 10968 (2019).

[79] J. Kalt, M. Sternik, I. Sergueev, J. Herfort, B. Jenichen, H.-C. Wille, O. Sikora, P. Piekarz, K. Parlinski, T. Baumbach, and S. Stankov, Phys. Rev. B 98, 121409(R) (2018).

[80] T. Ślęzak, J. Łażewski, S. Stankov, K. Parlinski, R. Reitinger, M. Rennhofer, R. Rüffer, B. Sepiol, M. Ślęzak, N. Spiridis, M. Zając, A. I. Chumakov, and J. Korecki, Phys. Rev. Lett. 99, 066103 (2007).

[81] S. Stankov, R. Röhlsberger, T. Ślęzak, M. Sladecek, B. Sepiol, G. Vogl, A. I. Chumakov, R. Rüffer, N. Spiridis, J. Łażewski, K. Parliński, and J. Korecki, Phys. Rev. Lett. 99, 185501 (2007).

[82] M. Y. Hu, W. Sturhahn, T. S. Toellner, P. D. Mannheim, D. E. Brown, J. Zhao, and E. E. Alp, Phys. Rev. B 67, 094304 (2003). 